\title{
Prevalence of High-Burden Medical Conditions Among Young and Middle-Aged Adults With Pediatric-Onset Medical Conditions: Findings From US Private and Public Administrative Claims Data
}

\author{
Daniel G. Whitney ${ }^{*} \mathbb{D}$
}

\begin{abstract}
Adults with pediatric-onset medical conditions (POMCs) are susceptible to early development of high-burden medical conditions. However, research pertaining to this topic is lacking, which is vital information that could assist in health benefit planning and administration. The purpose of this study was to determine the prevalence of high-burden medical conditions among privately and publicly insured adults with POMCs, as compared to adults without POMCs, from the US. Data from 2016 were extracted from Optum Clinformatics ${ }^{\circledR}$ Data Mart (private insurance) and a random $20 \%$ sample from Medicare fee-for-service (public insurance). International Classification of Diseases, Tenth Revision, Clinical Modification (ICD-10-CM) diagnosis codes were used to identify 18-64-year-old beneficiaries with POMCs, as well as several high-burden medical conditions, including pain, fracture, mood affective disorders, anxiety disorders, ischemic heart diseases, cerebrovascular diseases, hypertensive and other cardiovascular diseases, type 2 diabetes, osteoporosis, osteoarthritis, chronic obstructive pulmonary diseases, liver diseases, and cancer. Privately and publicly insured adults with POMCs had higher prevalence of all medical conditions compared to adults without POMCs. Publicly insured adults with POMCs had higher prevalence of all medical conditions compared to privately insured adults with POMCs, except for the lower prevalence of pain and cancer. When stratified by the category of POMCs (eg, musculoskeletal, circulatory), privately and publicly insured groups tended to have higher prevalence of most (private) or all (public) medical conditions compared to adults without POMCs. Adults with POMCs have higher prevalence of several highburden medical conditions compared to adults without POMCs. This health disparity was present regardless of insurance coverage, but was generally more pronounced for public vs. private insured adults with POMCs.

Keywords: Clinical Epidemiology, Pediatric-Onset Medical Conditions, Noncommunicable Diseases, United States Copyright: @ 2019 The Author(s); Published by Kerman University of Medical Sciences. This is an open-access article distributed under the terms of the Creative Commons Attribution License (http://creativecommons.org/licenses/ by/4.0), which permits unrestricted use, distribution, and reproduction in any medium, provided the original work is properly cited.

Citation: Whitney DG. Prevalence of high-burden medical conditions among young and middle-aged adults with pediatric-onset medical conditions: findings from US private and public administrative claims data. Int J Health Policy Manag. 2019;8(11):629-635. doi:10.15171/ijhpm.2019.62
\end{abstract}

Article History:

Received: 27 March 2019 Accepted: 15 July 2019 ePublished: 29 July 2019

\section{Background}

Noncommunicable diseases are growing public health issues, ${ }^{1}$ and represent a considerable source for early mortality ${ }^{2}$ and economic burden. ${ }^{3}$ The burden of noncommunicable diseases may disproportionally affect populations with disabilities, especially if the disability originated in childhood.

Pediatric-onset medical conditions (POMCs) refer to a group of congenital or acquired conditions that originate at any point from conception through adolescence, and consist of impairments in behavioral, emotional, mental, intellectual, or physical function, or are associated with altered development or abnormal physiological processes. Very little clinical and research attention has been given to individuals with POMCs as they become adults, despite the anticipated growth of the adult population with POMCs. ${ }^{4}$ Importantly, individuals with POMCs have complicated healthcare needs throughout the lifespan. This increases risk for early development of highburden medical conditions, which refer to conditions that lead to high financial cost, excess morbidity, early mortality, or poor quality of life, and include pain, fracture, and noncommunicable diseases. ${ }^{5-8}$ Therefore, a comprehensive understanding of the clinical epidemiology of high-burden medical conditions among young and middle-aged adults with POMCs is needed. Such information could inform healthcare policy-makers and clinical practice guidelines that promote early and cost-effective care for the aging population with POMCs. This study sought to inform these efforts by providing recent US national level estimates of the prevalence of several high-burden medical conditions for adults with POMCs that have private or public health insurance, as compared to adults without POMCs. We hypothesized that adults with POMCs would have higher prevalence of all high-burden medical conditions compared to adults without POMCs. We also hypothesized that this disparity would be greater for publicly insured adults with POMCs than privately insured adults with POMCs. 


\section{Methods}

\section{Data Sources}

Data for this study were extracted from private and public administrative claims data from the year 2016. Optum Clinformatics $^{\circledR}$ Data Mart Database (OptumInsight ${ }^{\mathrm{TM}}$, Eden Prairie, MN, USA) provided de-identified information for privately insured beneficiaries (commercial or Medicare Advantage plans). A random 20\% sample of the Medicare feefor-service database provided de-identified information for publicly insured beneficiaries.

\section{Sample Selection}

Beneficiaries that were 18 to 64 years of age, had 12 full months of continuous enrollment, and had at least one medical service utilization in 2016 were included. Medicare beneficiaries were excluded if they were covered by Health Maintenance Organization plans because of incomplete claims. Medical diagnoses of all POMCs and medical conditions were identified using the International Classification of Diseases, Tenth Revision, Clinical Modification (ICD-10-CM) codes, and are presented in Table S1 (see Supplementary file 1).

A single claim in any position (medical file in Optum; inpatient, outpatient, and carrier file in Medicare) for POMCs was used to identify these beneficiaries. The single claimbased definition has shown good accuracy for identifying pediatric-onset conditions using administrative claims data, with sensitivity of $99 \%$ and positive predictive value of $79 \% .{ }^{9}$ It is not possible to determine if some POMCs developed in childhood or adulthood (eg, spinal cord injury) using a single year of claims data. Therefore, only conditions with known childhood origins were included. These conditions were then grouped into the following POMC subcategories: musculoskeletal system; neurodevelopmental; circulatory system; nervous system; genital organs; other chromosomal abnormalities; urinary system; respiratory and digestive systems; and malformations of the eye, ear, face, and neck. Beneficiaries of private insurance without any medical claims for POMCs represented the group without POMCs. The group without POMCs were ascertained through Optum to enhance representativeness of the sample of adults without POMCs, because criteria for enrollment in Medicare under 65 years of age requires permanent disability.

\section{Medical Conditions}

Prevalent medical conditions were ascertained using the same procedure to identify POMCs. Medical conditions were selected with guidance from the literature on disease, disability, and mortality, ${ }^{1,2,5,10-12}$ and included pain, all-cause fracture, and several noncommunicable diseases: mood affective disorders; anxiety disorders; ischemic heart diseases; cerebrovascular diseases; hypertensive and other cardiovascular diseases; type 2 diabetes mellitus; osteoporosis; osteoarthritis; chronic obstructive pulmonary diseases; chronic kidney diseases; liver diseases; and malignant cancer anywhere in the body.

\section{Participant Characteristics}

Data on age and gender were extracted as they were reported in the same manner from Optum and Medicare. Other confounding variables were not considered for covariate adjustment to limit bias for reasons such as they had not been reported in both data sources (eg, education level), they had not been reported in the same manner (eg, race), or they had missing data on over $20 \%$ of the group (eg, income).

\section{Statistical Analysis}

All measures were summarized using SAS version 9.4 (SAS Institute, Inc., Cary, NC). Data were presented for adults without POMCs and with POMCs stratified by insurance coverage (private, public). Then, data were presented for adults with POMCs after stratifying by each POMC category for private and public insured beneficiaries. 95\% CIs were calculated for the POMC groups. A sensitivity analysis was performed to determine prevalence estimates of medical conditions, but with the requirement of having at least 2 claims from separate days for each POMC and medical condition category.

\section{Results}

Participant characteristics for adults without POMCs $(\mathrm{n}=5415475)$ and adults with POMCs that had private $(\mathrm{n}=121446)$ or public $(\mathrm{n}=119654)$ insurance is presented in Table 1. There were differences in the prevalence of POMC categories between private and public insurance. Notably, the publicly insured group had considerably ( $>3$ times) higher prevalence of neurodevelopmental POMCs and considerably (>3 times) lower prevalence of POMCs of the genital organs compared to the privately insured group.

Prevalence of high-burden medical conditions among adults with and without POMCs is presented in Table 2. Privately and publicly insured adults with POMCs had higher prevalence of all medical conditions compared to adults without POMCs. Publicly insured adults with POMCs had higher prevalence of all medical conditions compared to privately insured adults with POMCs, except for the lower prevalence of pain and cancer.

Prevalence of high-burden medical conditions for each of the 9 POMC categories is presented in Table 3. Compared to adults without POMCs, most categories of POMCs from the privately insured group (7 to 9 categories) tended to have higher prevalence of all medical conditions. Compared to adults without POMCs, most categories of POMCs from the publicly insured group ( 8 to 9 categories) tended to have higher prevalence of all medical conditions. Finally, compared to the privately insured group of adults with POMCs, the publicly insured group had higher prevalence of all high-burden medical conditions for each POMC category, except for the similar or lower prevalence between neurodevelopmental POMCs for cerebrovascular diseases, POMCs of the nervous system for pain, ischemic heart diseases, cerebrovascular diseases, liver diseases, and cancer, and POMCs of other chromosomal abnormalities for pain, liver diseases, and cancer.

The results of the sensitivity analysis requiring at least two claims from different days for each category of POMCs and medical conditions are presented in Table S2 for adults with and without POMCs and Table S3 after stratifying by the type 
Table 1. Characteristics and Prevalence of High-Burden Medical Conditions Among Adults With and Without POMCs

\begin{tabular}{|c|c|c|c|}
\hline & Without POMCs & With POMCs & With POMCs \\
\hline & $\begin{array}{l}\text { Private Insurance } \\
\text { (n= } 5415 \text { 475) }\end{array}$ & $\begin{array}{l}\text { Private Insurance } \\
\quad(n=121446)\end{array}$ & $\begin{array}{c}\text { Public Insurance } \\
\text { (n= } 119654)\end{array}$ \\
\hline Descriptive characteristics & Point estimate & Point estimate & Point estimate \\
\hline Age, mean (SD) & $43.8(12.9)$ & $44.3(13.6)$ & 46.9 (11.9) \\
\hline $18-40$ years, $\%$ & 40.3 & 44.1 & 30.9 \\
\hline $41-64$ years, $\%$ & 59.7 & 55.9 & 69.1 \\
\hline \multicolumn{4}{|l|}{ Gender, \% } \\
\hline Female & 53.8 & 55.6 & 46.8 \\
\hline Male & 46.2 & 44.4 & 53.2 \\
\hline \multicolumn{4}{|l|}{ POMC categories, \% } \\
\hline Musculoskeletal system & & 27.6 & 10.8 \\
\hline Neurodevelopmental & & 14.1 & 56.7 \\
\hline Circulatory system & & 20.1 & 9.4 \\
\hline Nervous system & & 9.1 & 18.6 \\
\hline Genital organs & & 5.1 & 0.9 \\
\hline Other chromosomal abnormalities & & 4.5 & 9.6 \\
\hline Urinary system & & 10.3 & 5.9 \\
\hline Respiratory and digestive system & & 10.0 & 4.0 \\
\hline Malformation of eye, ear, face, or neck & & 4.8 & 2.1 \\
\hline
\end{tabular}

Abbreviations: POMCs, pediatric-onset medical conditions; SD, standard deviation.

Note: Individuals may have more than one POMC and can be represented across multiple POMC categories.

Table 2. Prevalence of High-Burden Medical Conditions Among Adults With and Without POMCs

\begin{tabular}{|c|c|c|c|}
\hline & Without POMCs & With POMCs & With POMCs \\
\hline & $\begin{array}{l}\text { Private Insurance } \\
\text { (n = 5415475) }\end{array}$ & $\begin{array}{l}\text { Private Insurance } \\
(n=121446)\end{array}$ & $\begin{array}{c}\text { Public Insurance } \\
\text { (n = 119654) }\end{array}$ \\
\hline & $\%$ & $\%(95 \% \mathrm{Cl})$ & $\%(95 \% \mathrm{Cl})$ \\
\hline Pain & 30.1 & $45.2(44.9,45.5)$ & $42.6(42.3,42.9)$ \\
\hline Fracture & 2.6 & $5.2(5.1,5.3)$ & $7.8(7.6,8.0)$ \\
\hline Anxiety disorders & 15.1 & $25.5(25.3,25.7)$ & $37.9(37.6,38.2)$ \\
\hline Ischemic heart diseases & 3.5 & $8.7(8.5,8.9)$ & $11.4(11.2,11.6)$ \\
\hline Cerebrovascular diseases & 1.5 & $6.3(6.2,6.4)$ & $8.6(8.4,8.8)$ \\
\hline Hypertensive and other cardiovascular diseases & 25.4 & $37.8(37.5,38.1)$ & $50.6(50.3,50.9)$ \\
\hline Type 2 diabetes & 10.0 & $14.5(14.3,14.7)$ & $24.9(24.7,25.1)$ \\
\hline Osteoporosis & 1.3 & $3.4(3.3,3.5)$ & $7.4(7.3,7.5)$ \\
\hline Chronic obstructive pulmonary disease & 0.7 & $1.8(1.7,1.9)$ & $12.0(11.8,12.2)$ \\
\hline Chronic kidney disease & 1.8 & $6.6(6.5,6.7)$ & $11.4(11.2,11.6)$ \\
\hline Liver diseases & 2.9 & $7.3(7.2,7.4)$ & $8.2(8.0,8.4)$ \\
\hline Cancer & 3.8 & $6.6(6.5,6.7)$ & $5.9(5.8,6.0)$ \\
\hline
\end{tabular}

Abbreviation: POMCs, pediatric-onset medical conditions.

of POMC category (see Supplementary file 1). Interpretations were unchanged for adults with and without POMCs. Interpretations were largely unchanged for most categories of POMCs from the privately and publicly insured group compared to controls.

\section{Discussion}

The chief finding here is that young and middle-aged adults with POMCs had higher prevalence of several high-burden medical conditions compared to adults without POMCs. While this was evident for both privately and publicly insured adults with POMCs, these health disparities were more pronounced for the publicly insured group with POMCs. These are critical findings, because these high-burden medical conditions are major contributors to the global and national burden of disease and mortality, ${ }^{2,13-15}$ and are typically associated with advanced aging. Further, many of these high-burden medical conditions can be prevented, delayed, or better managed to 


\begin{tabular}{|c|c|c|c|c|c|c|c|c|c|}
\hline & Musculoskeletal System & Neurodevelopmental & Circulatory System & Nervous System & Genital Organs & $\begin{array}{l}\text { Chromosomal } \\
\text { Abnormalities }\end{array}$ & Urinary System & $\begin{array}{l}\text { Respiratory Digestive } \\
\text { Systems }\end{array}$ & Eye, Ear, Face, or Neck \\
\hline \multicolumn{10}{|l|}{ Sample size, $\mathrm{n}$} \\
\hline Private insurance & 33566 & 17149 & 24393 & 11021 & 6164 & 5518 & 12477 & 12190 & 5811 \\
\hline Public insurance & 12910 & 67791 & 11276 & 22276 & 1070 & 11431 & 6998 & 4744 & 2454 \\
\hline \multicolumn{10}{|l|}{ Pain, \% (95\% Cl) } \\
\hline Private insurance & $63.4(62.9,63.9)$ & $27.8(27.1,28.5)$ & $39.8(39.2,40.4)$ & $46.0(45.1,46.9)$ & $31.9(30.7,33.1)$ & $27.2(26.0,28.4)$ & $47.3(46.4,48.2)$ & $45.3(44.4,46.2)$ & $34.4(33.2,35.6)$ \\
\hline \multicolumn{10}{|l|}{ Fracture, \% (95\% Cl) } \\
\hline Private insurance & $7.7(7.4,8.0)$ & $4.8(4.5,5.1)$ & $4.1(3.9,4.3)$ & $5.7(5.3,6.1)$ & $2.3(1.9,2.7)$ & $3.6(3.1,4.1)$ & $4.5(4.1,4.9)$ & $4.7(4.3,5.1)$ & $3.5(3.0,4.0)$ \\
\hline Public insurance & $12.2(11.6,12.8)$ & $7.3(7.1,7.5)$ & $8.6(8.1,9.1)$ & $7.5(7.2,7.8)$ & $7.9(6.3,9.5)$ & $5.2(4.8,5.6)$ & $9.0(8.3,9.7)$ & $10.1(9.2,11.0)$ & $7.5(6.5,8.5)$ \\
\hline \multicolumn{10}{|c|}{ Mood affective disorders, \% (95\% Cl) } \\
\hline Private insurance & $19.2(18.8,19.6)$ & $35.4(34.7,36.1)$ & $18.3(17.8,18.8)$ & $24.7(23.9,25.5)$ & $13.5(12.6,14.4)$ & $13.7(12.8,14.6)$ & $18.8(18.1,19.5)$ & $20.6(19.9,21.3)$ & $14.5(13.6,15.4)$ \\
\hline Public insurance & $44.2(43.3,45.1)$ & $45.1(44.7,45.5)$ & $39.7(38.8,40.6)$ & $30.8(30.2,31.4)$ & $45.4(42.4,48.4)$ & $22.1(21.3,22.9)$ & $39.8(38.7,40.9)$ & $48.7(47.3,50.1)$ & $34.4(32.5,36.3)$ \\
\hline Private insurance & $24.2(23.7,24.7)$ & $37.0(36.3,37.7)$ & $24.1(23.6,24.6)$ & $27.1(26.3,27.9)$ & $20.1(19.1,21.1)$ & $18.7(17.7,19.7)$ & $23.6(22.9,24.3)$ & $26.6(25.8,27.4)$ & $19.0(18.0,20.0)$ \\
\hline Public insurance & $42.8(41.9,43.7)$ & $39.9(39.5,40.3)$ & $39.8(38.9,40.7)$ & $29.0(28.4,29.6)$ & $44.2(41.2,47.2)$ & $23.0(22.2,23.8)$ & $39.8(38.7,40.9)$ & $49.4(48.0,50.8)$ & $31.8(30.0,33.6)$ \\
\hline \multicolumn{10}{|c|}{ Ischemic heart diseases, \% (95\% CI) } \\
\hline Private insurance & $5.4(5.2,5.6)$ & $4.7(4.4,5.0)$ & $19.9(19.4,20.4)$ & $5.6(5.2,6.0)$ & $2.9(2.5,3.3)$ & $3.2(2.7,3.7)$ & $10.9(10.4,11.4)$ & $8.4(7.9,8.9)$ & $4.4(3.9,4.9)$ \\
\hline Public insurance & $14.7(14.1,15.3)$ & $6.8(6.6,7.0)$ & $33.8(32.9,34.7)$ & $5.8(5.5,6.1)$ & $14.5(12.4,16.6)$ & $4.4(4.0,4.8)$ & $26.9(25.9,27.9)$ & $21.7(20.5,22.9)$ & $11.1(9.9,12.3)$ \\
\hline \multicolumn{10}{|c|}{ Cerebrovascular diseases, \% (95\% Cl) } \\
\hline Private insurance & $3.1(2.9,3.3)$ & $6.2(5.8,6.6)$ & $15.0(14.6,15.4)$ & $7.9(7.4,8.4)$ & $1.7(1.4,2.0)$ & $2.8(2.4,3.2)$ & $5.5(5.1,5.9)$ & $4.0(3.7,4.3)$ & $2.9(2.5,3.3)$ \\
\hline Public insurance & $9.0(8.5,9.5)$ & $6.2(6.0,6.4)$ & $24.7(23.9,25.5)$ & $7.7(7.3,8.1)$ & $8.6(6.9,10.3)$ & $4.3(3.9,4.7)$ & $13.7(12.9,14.5)$ & $11.9(11.0,12.8)$ & $6.1(5.2,7.0)$ \\
\hline \multicolumn{10}{|c|}{ Hypertensive and other cardiovascular diseases, \% (95\% Cl) } \\
\hline Private insurance & $32.2(31.7,32.7)$ & $32.3(31.6,33.0)$ & $49.0(48.4,49.6)$ & $35.8(34.9,36.7)$ & $19.5(18.5,20.5)$ & $21.1(20.0,22.2)$ & $57.7(56.8,58.6)$ & $40.1(39.2,41.0)$ & $28.8(27.6,30.0)$ \\
\hline Public insurance & $59.3(58.5,60.1)$ & $45.4(45.0,45.8)$ & $71.4(70.6,72.2)$ & $42.4(41.8,43.0)$ & $58.6(55.6,61.6)$ & $28.8(28.0,29.6)$ & $83.4(82.5,84.3)$ & $67.2(65.9,68.5)$ & $50.3(48.3,52.3)$ \\
\hline \multicolumn{10}{|c|}{ Type 2 diabetes, \% (95\% Cl) } \\
\hline Private insurance & $12.9(12.5,13.3)$ & $16.7(16.1,17.3)$ & $15.7(15.2,16.2)$ & $12.7(12.1,13.3)$ & $7.7(7.0,8.4)$ & $11.7(10.9,12.5)$ & $18.9(18.2,19.6)$ & $15.1(14.5,15.7)$ & $14.3(13.4,15.2)$ \\
\hline
\end{tabular}




\section{Table 3. Continued}

\begin{tabular}{|c|c|c|c|c|c|c|c|c|c|}
\hline & Musculoskeletal System & Neurodevelopmental & Circulatory System & Nervous System & Genital Organs & $\begin{array}{l}\text { Chromosomal } \\
\text { Abnormalities }\end{array}$ & Urinary System & $\begin{array}{l}\text { Respiratory Digestive } \\
\text { Systems }\end{array}$ & Eye, Ear, Face, or Neck \\
\hline \multicolumn{10}{|c|}{ Osteoporosis, \% (95\% Cl) } \\
\hline Private insurance & $4.0(3.8,4.2)$ & $3.1(2.8,3.4)$ & $2.9(2.7,3.1)$ & $4.3(3.9,4.7)$ & $1.0(0.8,1.2)$ & $3.6(3.1,4.1)$ & $3.6(3.3,3.9)$ & $4.4(4.0,4.8)$ & $2.2(1.8,2.6)$ \\
\hline Public insurance & $11.0(10.5,11.5)$ & $7.3(7.1,7.5)$ & $7.4(6.9,7.9)$ & $10.9(10.5,11.3)$ & $6.7(5.2,8.2)$ & $7.5(7.0,8.0)$ & $8.0(7.4,8.6)$ & $10.5(9.6,11.4)$ & $7.1(6.1,8.1)$ \\
\hline \multicolumn{10}{|c|}{ Osteoarthritis, \% (95\% Cl) } \\
\hline Private insurance & $25.1(24.6,25.6)$ & $9.4(9.0,9.8)$ & $13.6(13.2,14.0)$ & $14.1(13.5,14.7)$ & $6.0(5.4,6.6)$ & $7.6(6.9,8.3)$ & $15.9(15.3,16.5)$ & $15.9(15.3,16.5)$ & $9.8(9.0,10.6)$ \\
\hline Public insurance & $40.0(39.2,40.8)$ & $13.7(13.4,14.0)$ & $26.0(25.2,26.8)$ & $15.5(15.0,16.0)$ & $26.5(23.9,29.1)$ & $12.2(11.6,12.8)$ & $29.2(28.1,30.3)$ & $32.5(31.2,33.8)$ & $20.3(18.7,21.9)$ \\
\hline \multicolumn{10}{|c|}{ Chronic obstructive pulmonary disease, \% (95\% Cl) } \\
\hline Private insurance & $1.4(1.3,1.5)$ & $1.2(1.0,1.4)$ & $2.6(2.4,2.8)$ & $1.6(1.4,1.8)$ & $0.7(0.5,0.9)$ & $0.9(0.7,1.1)$ & $2.4(2.1,2.7)$ & $2.7(2.4,3.0)$ & $1.1(0.8,1.4)$ \\
\hline Public insurance & $18.0(17.3,18.7)$ & $8.6(8.4,8.8)$ & $24.0(23.2,24.8)$ & $7.5(7.2,7.8)$ & $15.1(13.0,17.2)$ & $4.5(4.1,4.9)$ & $21.1(20.1,22.1)$ & $27.1(25.8,28.4)$ & $11.5(10.2,12.8)$ \\
\hline \multicolumn{10}{|c|}{ Chronic kidney disease, \% (95\% Cl) } \\
\hline Private insurance & $3.2(3.0,3.4)$ & $5.1(4.8,5.4)$ & $6.4(6.1,6.7)$ & $4.3(3.9,4.7)$ & $2.3(1.9,2.7)$ & $4.0(3.5,4.5)$ & $27.7(26.9,28.5)$ & $5.3(4.9,5.7)$ & $3.3(2.8,3.8)$ \\
\hline Public insurance & $11.6(11.0,12.2)$ & $6.7(6.5,6.9)$ & $21.5(20.7,22.3)$ & $5.7(5.4,6.0)$ & $15.5(13.3,17.7)$ & $6.8(6.3,7.3)$ & $57.2(56.0,58.4)$ & $18.2(17.1,19.3)$ & $11.3(10.0,12.6)$ \\
\hline \multicolumn{10}{|c|}{ Liver diseases, \% (95\% Cl) } \\
\hline Private insurance & $4.9(4.7,5.1)$ & $4.3(4.0,4.6)$ & $7.5(7.2,7.8)$ & $4.6(4.2,5.0)$ & $4.0(3.5,4.5)$ & $4.6(4.0,5.2)$ & $17.2(16.5,17.9)$ & $15.4(14.8,16.0)$ & $4.3(3.8,4.8)$ \\
\hline Public insurance & $10.1(9.6,10.6)$ & $5.8(5.6,6.0)$ & $13.5(12.9,14.1)$ & $5.2(4.9,5.5)$ & $12.7(10.7,14.7)$ & $5.0(4.6,5.4)$ & $22.1(21.1,23.1)$ & $22.9(21.7,24.1)$ & $7.1(6.1,8.1)$ \\
\hline \multicolumn{10}{|l|}{ Cancer, \% (95\% Cl) } \\
\hline Private insurance & $5.2(5.0,5.4)$ & $3.5(3.2,3.8)$ & $7.1(6.8,7.4)$ & $5.4(5.0,5.8)$ & $4.6(4.1,5.1)$ & $5.7(5.1,6.3)$ & $11.9(11.3,12.5)$ & $10.5(10.0,11.0)$ & $6.6(6.0,7.2)$ \\
\hline Public insurance & $7.7(7.2,8.2)$ & $4.0(3.9,4.1)$ & $9.1(8.6,9.6)$ & $4.4(4.1,4.7)$ & $10.7(8.8,12.6)$ & $3.4(3.1,3.7)$ & $14.1(13.3,14.9)$ & $13.2(12.2,14.2)$ & $7.3(6.3,8.3)$ \\
\hline
\end{tabular}

Abbreviation: POMCs, pediatric-onset medical conditions.

Note: Individuals may have more than one POMC and can be represented across multiple POMC categories. 
reduce patient and caregiver burden. Taken together, these findings highlight the need for earlier clinical, rehabilitative, and healthcare policy efforts to improve awareness and access to quality healthcare, such as screening strategies, for these populations to allow for earlier detection of these highburden medical conditions.

In the current study, we also found that adults with POMCs of a particular biological system may be at greater risk of high-burden medical conditions of that same biological system. Notably, POMCs of the musculoskeletal system had the highest prevalence of fracture, osteoporosis, and osteoarthritis; POMCs of the circulatory system had the highest prevalence of ischemic heart diseases, cerebrovascular diseases, and hypertensive and other cardiovascular diseases; and POMCs of the urinary system had the highest prevalence of chronic kidney diseases. This information could assist clinical care for adults with POMCs, by allowing clinicians to target preventative and rehabilitation care specific to the needs of their patients with POMCs. This information could also assist in health benefit planning, by allowing administrators to allocate healthcare resources based on the POMC demographics of health plans.

A major strength of the current study is the large sample of adults with POMCs from both private and public insurance databases, thus enhancing external validity. ${ }^{16}$ Gathering data on clinical populations can be challenging, and little is known about the healthful aging process for individuals with POMCs across the lifespan. Another major strength of this study is the comprehensive assessment of several high-burden medical conditions, with many representing major contributors to the global and national burden of disease and mortality. ${ }^{2,13-15}$ The present findings are robust and could improve clinical care and inform new research examining health disparities and healthcare delivery for individuals with POMCs.

The limitations must be discussed. First, while Optum and Medicare databases are nationwide, Optum coverage is more predominant in Southern and Western regions of the United States, which may bias estimates because of regional differences in chronic disease prevalence. ${ }^{17}$ Second, the primary analysis used a single claim to identify beneficiaries with POMCs and medical conditions. While validation studies have shown that at least 2 claims for a medical condition improves ability to identify beneficiaries with that medical condition, ${ }^{9,18}$ single claim-based algorithms have been reported to have moderate-to-high positive predictive value $(\sim 80 \%)$ to detect POMCs, ${ }^{9}$ or specificity up to $96 \%$ to detect a variety of medical conditions; ${ }^{19,20}$ however, the accuracy of identifying conditions using claims data depends on the length of the study period ${ }^{19}$ and the medical condition examined., ${ }^{919-21}$ In light of this, a sensitivity analysis was performed that used at least two claims from different days to identify all POMCs and medical conditions. The results revealed the same conclusions as the primary analysis. When taken together, given the short study period of 12 months, the selected methodology to identify medical conditions using a single claim is likely sufficient to detect that the current data is evidence of health disparities among adults with POMCs, as compared to adults without POMCs. Third, the present study excluded individuals that did not have service utilization in 2016, which may have biased results. However, these excluded individuals that had insurance coverage may be somewhat healthy since they did not require a medical encounter in 2016, thus potentially biasing results in the present study to be more conservative estimates. Fourth, it is not possible using claimsbased data from a single year to determine if certain conditions developed in childhood or later in life (eg, spinal cord injury, cancer, depression). Fifth, data were from a single year and longer study periods and longitudinal research designs could provide more robust findings and implications for research, practice, and policy. Lastly, the present study presented descriptive statistics rather than inferential statistics, and therefore did not account for potential confounding factors, such as indicators of socioeconomic status.

\section{Conclusion}

Adults with POMCs have higher prevalence of several high-burden medical conditions compared to adults without POMCs. This health disparity was present for both private and public insurance coverage, but was generally more pronounced for public vs. private insured adults with POMCs. Study findings are critical, because many of these high-burden medical conditions are associated with advanced aging, and may be prevented, delayed, or better managed with adequate clinical knowledge. These findings highlight the need for clinical and public health strategies to implement earlier detection and preventive medical services for noncommunicable diseases and other high-burden medical conditions for adult populations with POMCs. Future research is needed to examine how these high-burden medical condition profiles associate with mortality and quality of life for adults with POMCs.

\section{Ethical issues}

Since data are de-identified, consent to participate was not required and the University IRB approved this study as non-regulated.

\section{Competing interests}

Author declares that he has no competing interests.

Author's contribution

DGW is the single author of the paper.

\section{Supplementary files}

Supplementary file 1 contains Tables S1-S3.

\section{References}

1. Global, regional, and national disability-adjusted life-years (DALYs) for 359 diseases and injuries and healthy life expectancy (HALE) for 195 countries and territories, 1990-2017: a systematic analysis for the Global Burden of Disease Study 2017. Lancet. 2018;392(10159):1859-1922. doi:10.1016/S0140-6736(18)32335-3

2. Global, regional, and national age-sex specific mortality for 264 causes of death, 1980-2016: a systematic analysis for the Global Burden of Disease Study 2016. Lancet. 2017;390(10100):11511210. doi:10.1016/S0140-6736(17)32152-9

3. WHO. Global action plan for the prevention and control of noncommunicable diseases 2013-2020. Geneva, Switzerland: World Health Organization;2013.

4. Global Research on Developmental Disabilities C. Developmental disabilities among children younger than 5 years in 195 countries and territories, 1990-2016: a systematic analysis for the Global Burden of Disease Study 2016. Lancet Glob Health. 2018. 
doi:10.1016/S2214-109X(18)30309-7

5. Whitney DG, Hurvitz EA, Ryan JM, et al. Noncommunicable disease and multimorbidity in young adults with cerebral palsy. Clin Epidemiol. 2018;10:511-519. doi:10.2147/CLEP.S159405

6. Whitney DG, Shapiro DN, Warschausky SA, Hurvitz EA, Peterson MD. The contribution of neurologic disorders to the national prevalence of depression and anxiety problems among children and adolescents. Ann Epidemiol. 2018. doi:10.1016/j. annepidem.2018.11.003

7. Ryan JM, Allen E, Gormley J, Hurvitz EA, Peterson MD. The risk, burden, and management of non-communicable diseases in cerebral palsy: a scoping review. Dev Med Child Neurol. 2018;60(8):753764. doi:10.1111/dmcn.13737

8. Charlson FJ, Baxter AJ, Dua T, Degenhardt L, Whiteford HA, Vos T. Mental, Neurological, and Substance Use Disorders: Disease Control Priorities, Third Edition. Chapter 3 Excess Mortality from Mental, Neurological, and Substance Use Disorders in the Global Burden of Disease Study 2010. Vol 4. Washington DC: The World Bank; 2016.

9. Reeves S, Garcia E, Kleyn M, et al. Identifying sickle cell disease cases using administrative claims. Acad Pediatr. 2014;14(5 Suppl):S61-67. doi:10.1016/j.acap.2014.02.008

10. Quinones AR, Markwardt S, Botoseneanu A. Multimorbidity Combinations and Disability in Older Adults. J Gerontol A Biol Sci Med Sci. 2016;71(6):823-830. doi:10.1093/gerona/glw035

11. Whitney DG, Hurvitz EA, Devlin MJ, et al. Age trajectories of musculoskeletal morbidities in adults with cerebral palsy. Bone. 2018;114:285-291. doi:10.1016/j.bone.2018.07.002

12. Burge R, Dawson-Hughes B, Solomon DH, Wong JB, King A, Tosteson A. Incidence and economic burden of osteoporosis-related fractures in the United States, 2005-2025. J Bone Miner Res. 2007;22(3):465-475. doi:10.1359/jbmr.061113

13. Murray CJ, Vos T, Lozano R, et al. Disability-adjusted life years (DALYs) for 291 diseases and injuries in 21 regions, 1990-2010: a systematic analysis for the Global Burden of Disease Study 2010. Lancet. 2012;380(9859):2197-2223. doi:10.1016/S01406736(12)61689-4

14. Whiteford HA, Degenhardt L, Rehm J, et al. Global burden of disease attributable to mental and substance use disorders: findings from the Global Burden of Disease Study 2010. Lancet. 2013;382(9904):1575-1586. doi:10.1016/S0140-6736(13)61611-6

15. Global, regional, and national incidence, prevalence, and years lived with disabilitity for 354 diseases and injuries for 195 countries and territories, 1990-2017: a systematic analysis for the Global Burden of Disease Study 2017. Lancet. 2018;392(10159):1789-1858.

16. Whitney DG, Alford AI, Devlin MJ, Caird MS, Hurvitz EA, Peterson $\mathrm{MD}$. Adults with cerebral palsy have higher prevalence of fracture compared to adults without cerebral palsy independent of osteoporosis and cardiometabolic diseases. J Bone Miner Res. 2019. doi:10.1002/jbmr.3694

17. Raghupathi W, Raghupathi V. An Empirical Study of Chronic Diseases in the United States: A Visual Analytics Approach. Int J Environ Res Public Health. 2018;15(3). doi:10.3390/ijerph15030431

18. Kerr EA, McGlynn EA, Van Vorst KA, Wickstrom SL. Measuring antidepressant prescribing practice in a health care system using administrative data: implications for quality measurement and improvement. Jt Comm J Qual Improv. 2000;26(4):203-216.

19. Leslie WD, Lix LM, Yogendran MS. Validation of a case definition for osteoporosis disease surveillance. Osteoporos Int. 2011;22(1):3746. doi:10.1007/s00198-010-1225-2

20. Doktorchik C, Patten S, Eastwood C, et al. Validation of a case definition for depression in administrative data against primary chart data as a reference standard. BMC Psychiatry. 2019;19(1):9. doi:10.1186/s12888-018-1990-6

21. Noyes K, Liu H, Lyness JM, Friedman B. Medicare beneficiaries with depression: comparing diagnoses in claims data with the results of screening. Psychiatr Serv. 2011;62(10):1159-1166. doi:10.1176/ ps.62.10.pss6210_1159 